\title{
On the choice of basis functions to model surface electric current densities in computational electromagnetics
}

\author{
Levent Gürel, Kubilay Sertel, ${ }^{1}$ and İbrahim Kürşat Şendur ${ }^{2}$ \\ Department of Electrical and Electronics Engineering, Bilkent University, Ankara, Turkey
}

\begin{abstract}
Basis functions that are used to model surface electric current densities in the electric field integral equations of computational electromagnetics are analyzed with respect to how well they model the charge distribution, in addition to the current. This analysis is carried out with the help of the topological properties of open and closed surfaces meshed into networks of triangles and quadrangles. The need for current basis functions to properly model the charge distribution is demonstrated by several examples. In some of these examples, the basis functions seem to be perfectly legitimate when only the current distribution is considered, but they fail to deliver a correct solution of the electromagnetic problem, since they are not capable of properly modeling the charge distribution on some surfaces. Although the idea of proper modeling of the charge distribution by the current basis functions is easy to accept and can even be claimed well known, the contrary uses encountered in the literature have been the motivation behind the investigation reported in this paper.
\end{abstract}

\section{Introduction}

Integral equation solution techniques, such as the method of moments (MOM) [Harrington, 1982; Miller et al., 1992], the fast multipole method (FMM) [Rokhlin, 1990; Coifman et al., 1993; Lu and Chew, 1993; Gürel and Aksun, 1996], and the recursive Tmatrix algorithms (RTMAs) [Chew et al., 1992; Gürel and Chew, 1993], express the unknown function using a set of known expansion functions or basis functions (BFs). Specifics of the problem and the solution technique may force or allow the BFs to be of very different forms.

\footnotetext{
${ }^{1}$ Now at the Radiation Laboratory, University of Michigan, Ann Arbor.

${ }^{2}$ Now at the Electroscience Laboratory, Ohio State University, Columbus.
}

Copyright 1999 by the American Geophysical Union.

Paper number 1999RS900008. 0048-6604/99/1999RS900008\$11.00
1. BFs may be vector or scalar functions, depending on the function they are modeling.

2. BFs may be entire-domain or subdomain functions, depending on their domain of definition [Kolundžija and Popović, 1993].

3. BFs may be curved or planar functions, depending on the surface on which they are defined [Wandzura, 1992; Valle et al., 1994; Song and Chew, 1995; Peterson and Aberegg, 1995].

4. BFs may be nodal, edge, or face functions, depending on which one they are associated with.

5. BFs may have linear, bilinear, quadratic, or higher-order variations [Wandzura, 1992; Aberegg et al., 1996; Graglia et al., 1997].

In addition to the BFs, another set of functions, called weighting functions or testing functions (TFs), are also employed in the solution of the integral equations. The choice of BFs and TFs has been investigated from many different points of view and reported by many researchers. Aksun and Mittra [1993] set forth conditions regarding the choice of BFs and TFs by considering the convergence of the integrals encountered in the spatial-domain and spectral-do- 
main formulations. Sarkar [1985] and Sarkar et al. [1985] discussed some of the mathematical restrictions on the choice of BFs by considering linear operators in general.

The merits of choosing the BFs identical to the TFs to obtain a Galerkin formulation and choosing the BFs as the complex conjugate of the TFs have also been discussed by many researchers [Sarkar, 1989; Richmond, 1991]. Among other consequences, the Galerkin formulation transforms the electric field integral equations (EFIEs) into symmetric systems of equations, which is an important consideration for the choice of the solution algorithm. Specific choices of BFs to create sparse systems of equations have also been proposed [Canning, 1993; Kim et al., 1996]. Andersson [1993] designed special BFs that include the singular behavior of the current to be modeled.

Furthermore, it is well known that for source-free and sink-free problems, the BFs should be chosen to make the integral of the charge distribution (total net charge) vanish. Continuity of the BFs in the direction of current flow must be assured to avoid any artificial and undesired charge accumulation.

All of the above are legitimate and important considerations in the choice of BFs. In addition to these, we will advocate one more constraint in this paper: The BFs chosen to model the electric current in the solution of an EFIE should also support a consistent and valid charge approximation. That is, the current $\mathrm{BFs}$ are responsible not only for the approximation of the current distribution, but also for the charge distribution. This is because the charge distribution is inherently and implicitly approximated by the divergences of the current BFs. This point will be further explained in section 2, and several examples of BFs satisfying and violating this constraint will be analyzed in section 4 . In section 3 , some relevant topological properties of surfaces will be summarized. We will need this information in order to relate the numbers of degrees of freedom (DOFs) supplied by the current approximation and required by the charge approximation.

\section{Modeling the Electric Current and Charge Distributions Together}

Solution of the EFIEs of the computational electromagnetics requires the expansion of the unknown current distribution $\mathbf{J}(\mathbf{r})$ in terms of the chosen basis functions. Using vector $\mathrm{BFs} \mathbf{b}_{n}(\mathbf{r})$, this expansion is given by

$$
\mathbf{J}(\mathbf{r})=\sum_{n=1}^{N} \mathbf{b}_{n}(\mathbf{r}) a_{n}
$$

using $N$ DOFs, where $a_{n}$ is a complex coefficient associated with the $\mathrm{BF} \mathbf{b}_{n}(\mathbf{r})$.

If scalar basis functions are employed, each vector component of $\mathbf{J}(\mathbf{r})$ can independently be expanded as

$$
\begin{aligned}
\mathbf{J}(\mathbf{r}) & =\sum_{n=1}^{N}\left[\begin{array}{ccc}
b_{n}^{(1)}(\mathbf{r}) & 0 & 0 \\
0 & b_{n}^{(2)}(\mathbf{r}) & 0 \\
0 & 0 & b_{n}^{(3)}(\mathbf{r})
\end{array}\right] \cdot\left[\begin{array}{c}
a_{n}^{(1)} \\
a_{n}^{(2)} \\
a_{n}^{(3)}
\end{array}\right] \\
& =\sum_{n=1}^{N} \overline{\mathbf{B}}_{n}(\mathbf{r}) \cdot \mathbf{a}_{n}
\end{aligned}
$$

using $3 N$ DOFs. Whether (1) or (2) is used for the modeling of the electric current distribution $\mathbf{J}(\mathbf{r})$, the electric charge density $\rho(\mathbf{r})$ is also being implicitly approximated by a collection of "charge BFs" without any need for an explicit implementation in the solution technique. Owing to the continuity equation (in the frequency domain with an assumed $e^{-i \omega t}$ time dependence)

$$
\nabla \cdot \mathbf{J}(\mathbf{r})=i \omega \rho(\mathbf{r})
$$

governing the relation between $\mathbf{J}(\mathbf{r})$ and $\rho(\mathbf{r})$, the charge distribution is implicitly modeled by the divergence of the BFs used to model the current distribution. Thus this implicit modeling of $\rho(\mathbf{r})$ corresponding to (1) and (2) is given by

$$
\begin{gathered}
\rho(\mathbf{r})=\frac{1}{i \omega} \sum_{n=1}^{N} \nabla \cdot \mathbf{b}_{n}(\mathbf{r}) a_{n} \\
\rho(\mathbf{r})=\frac{1}{i \omega} \sum_{n=1}^{N} \nabla \cdot \overline{\mathbf{B}}_{n}(\mathbf{r}) \cdot \mathbf{a}_{n}
\end{gathered}
$$

respectively, where

$$
\nabla \cdot \overline{\mathbf{B}}_{n}(\mathbf{r})=\left[\begin{array}{lll}
\frac{\partial b_{n}^{(1)}(\mathbf{r})}{\partial r_{1}} & \frac{\partial b_{n}^{(2)}(\mathbf{r})}{\partial r_{2}} & \frac{\partial b_{n}^{(3)}(\mathbf{r})}{\partial r_{3}}
\end{array}\right]_{(6)}
$$

and $r_{1}, r_{2}$, and $r_{3}$ are the three components of $\mathbf{r}$.

Since the charge distribution is expanded using the divergence of the current BFs and exactly the same coefficients are used in this expansion, we note the following:

1. The number of the DOFs used in the expansion of $\rho(\mathbf{r})$ in (5) and (6) is equal to the number of the 
DOFs supplied by the expansion of $\mathbf{J}(\mathbf{r})$ in (1) and (2). Note that this supplied number of DOFs may or may not be sufficient for a proper approximation of the charge distribution, and this is exactly what we will investigate in the remainder of this paper.

2. The nature of the expansion of $\rho(\mathbf{r})$ is determined by the kind of expansion chosen for $\mathbf{J}(\mathbf{r})$. For instance, if the current is approximated by entiredomain $\mathrm{BFs}$, then the charge is also approximated by entire-domain BFs. If the current is approximated by piecewise (PW) constant subdomain BFs, then the charge is approximated by singular functions defined on the subdomain boundaries ("blades") that coincide with the discontinuities of the normal component of the current. If the current is approximated by subdomain BFs that are PW linear and continuous along the direction of the current flow and PW constant in transverse directions, then the charge is approximated by a PW constant distribution. If a surface current is approximated by PW bilinear subdomain BFs that are continuous at least in the direction of the current flow, then the charge distribution is approximated by a PW linear distribution.

In this paper, we will focus on the use of the subdomain BFs for the approximation of the surface electric current densities. The use of the subdomain BFs fur the current results in the PW approximation of both the current and the charge on these subdomains. Note that, in general, the number of subdomains is not equal to the number of the BFs or the DOFs. Thus it is not certain that the DOFs used in the current expansion will be sufficient for the DOFs required by the charge distribution on all of the subdomains for the kind of approximation imposed by the choice of the current expansion.

In section 4, a class of BFs will be analyzed from this point of view. Before that, we will briefly study the topology of open and closed surfaces and their meshings into triangular and quadrangular networks, in section 3. We will need this information to compare the number of current BFs, which are related to the number of edges or nodes of a network, with the number of the required DOFs for the charge approximation, which is related to the number of faces of the same network.

\section{Topological Properties of Surfaces}

This section is intentionally kept as short as possible since this topic is extensively studied elsewhere.
For the purposes of this paper, statement of the results should be sufficient; derivations are not given.

The Euler's relation for open or closed, flat or curved surfaces containing $V$ vertices, $E$ edges, and $F$ faces states that

$$
V-E+F=\chi,
$$

where $\chi$ is called the Euler characteristic of the surface [Mortenson, 1985; Wandzura, 1992]. Here $\chi=0$ for a torus, $\chi=1$ for all simply connected open surfaces, and $\chi=2$ for all simple polyhedra that can be continuously deformed into a sphere. For example, a simple cube has eight vertices $(V=8)$, twelve edges $(E=12)$, and six faces $(F=6)$. Therefore the Euler characteristic of the surface of the cube is $\chi=8-12+6=2$. Let $V_{\text {ext }}, E_{\text {ext }}, V_{\text {int }}$, and $E_{\text {int }}$ denote the numbers of vertices and edges that are and are not on the exterior boundary of a surface. Then, we have the trivial equalities

$$
\begin{aligned}
& V=V_{\text {int }}+V_{\text {ext }}, \\
& E=E_{\text {int }}+E_{\text {ext }}
\end{aligned}
$$

and the obvious equality

$$
E_{\mathrm{ext}}=V_{\mathrm{ext}}
$$

where both are equal to zero for a closed surface. If the surface is meshed into a network of triangles or quadrangles, more relations can be found to relate $V, E$, and $F$.

\subsection{Quadrangular Networks}

Since each quadrangle has four edges and each internal edge is shared by two quadrangles, whereas each external edge belongs to only one quadrangle, we have

$$
4 F=2 E_{\text {int }}+E_{\text {ext }} .
$$

Here $2 E / V$ is defined as the average number of edges that meet at a vertex of a large quadrangular network since each edge terminates in two vertices. Combining (11) with (7)-(10), we can express this quantity as

$$
\frac{2 E}{V}=\frac{4 V_{\mathrm{int}}+3 V_{\mathrm{ext}}-4 \chi}{V} .
$$

\subsection{Triangular Networks}

Since each triangle has three edges and each internal edge is shared by two triangles, whereas each 
external edge belongs to only one triangle, we have

$$
3 F=2 E_{\text {int }}+E_{\text {ext }} .
$$

The above can be combined with (7)-(10) to derive the average number of edges that meet at a vertex of a large triangular network as

$$
\frac{2 E}{V}=\frac{6 V_{\mathrm{int}}+4 V_{\mathrm{ext}}-6 \chi}{V} .
$$

\section{Analysis of a Class of Basis Functions}

In this section we will consider several well-known subdomain BFs and their straightforward extensions to model the surface electric current densities. These

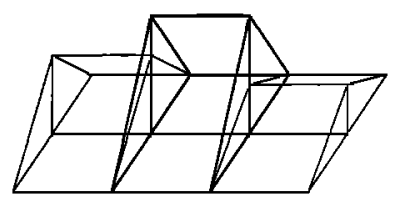

(a)

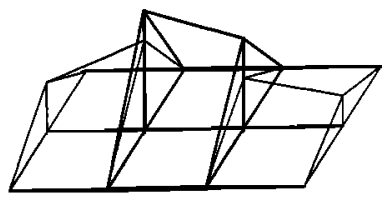

(b)

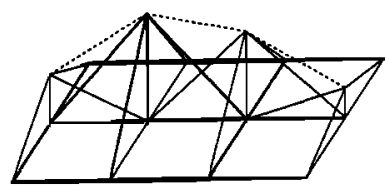

(d)

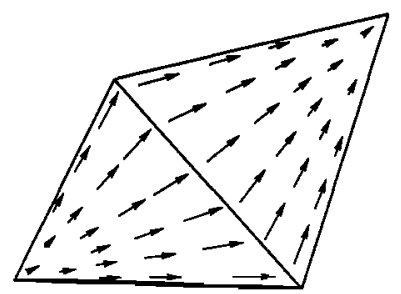

(f)

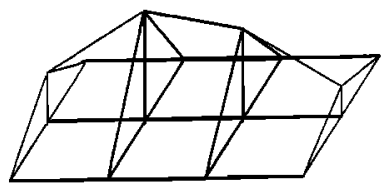

(c)

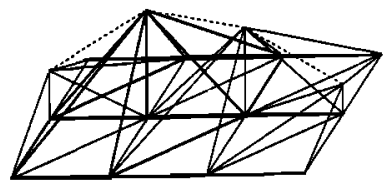

(e)

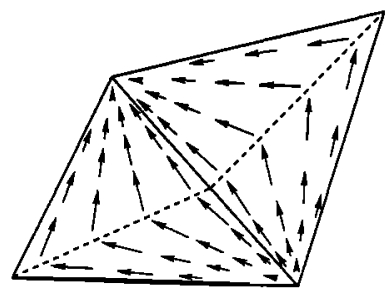

(g)
Figure 1. Basis functions (BFs) to be analyzed in this paper: (a) Rooftop (RT) BFs, (b) higherorder RT BFs, (c) transversely continuous higherorder RT BFs, (d) pyramidal BFs with rectangular bases, (e) pyramidal BFs with triangular bases, (f) Rao-Wilton-Glisson (RWG) BFs, and (g) Jacobus and Landstorfer's magnetic BFs.

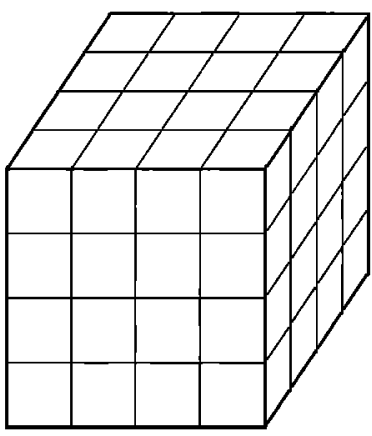

(a)

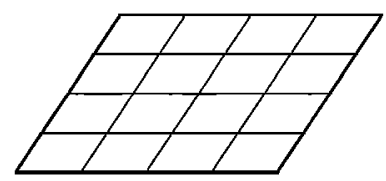

(c)

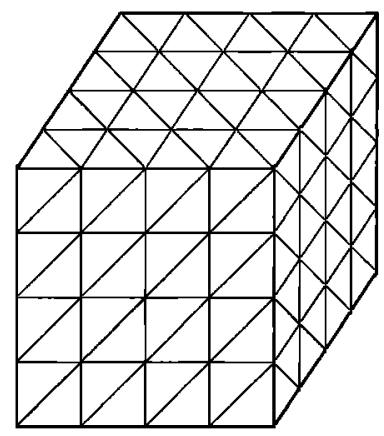

(b)

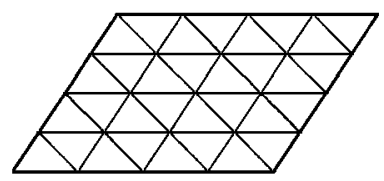

(d)
Figure 2. Examples of closed and open surfaces: (a) Rectangular prism meshed with quadrangles, (b) rectangular prism meshed with triangles, (c) flat patch meshed with quadrangles, and (d) flat patch meshed with triangles.

BFs are illustrated in Figure 1. We will investigate these BFs with the emphasis being on how well the charge distribution is modeled.

Using the topological properties summarized in the previous section, we will try to keep our results as general as possible for open and closed surfaces. When appropriate, rectangular prisms as in Figures $2 \mathrm{a}$ and $2 \mathrm{~b}$ and flat patches as in Figures 2c and $2 \mathrm{~d}$ will be used as examples of closed and open surfaces, respectively.

\subsection{Rooftop Basis Functions}

Rooftop (RT) BFs [Glisson and Wilton, 1980; Rubin and Daijavad, 1990] are defined on the internal edges of a quadrangular mesh. The total number of the DOFs is equal to $E_{\text {int }}$. Since the current approximation is $\mathrm{PW}$ linear and continuous along the direction of the current flow and PW constant in the transverse direction as shown in Figure 1a, the charge is approximated by a PW constant distribution. That is, with the choice of RT BFs for the current, the charge is allowed to take a constant value on each quadrangular subdomain. Therefore the number of DOFs required for the charge distribution is equal to $F$. 


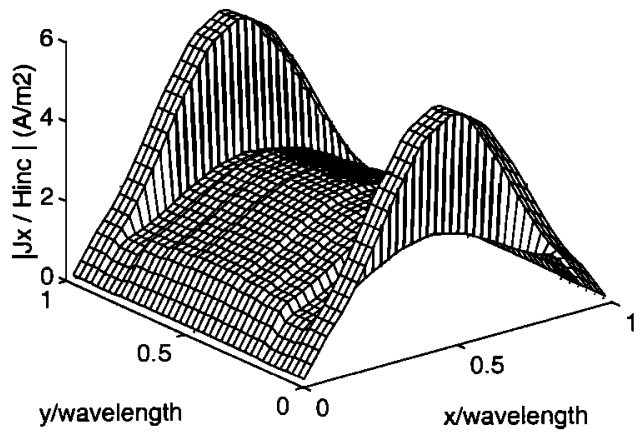

(a)

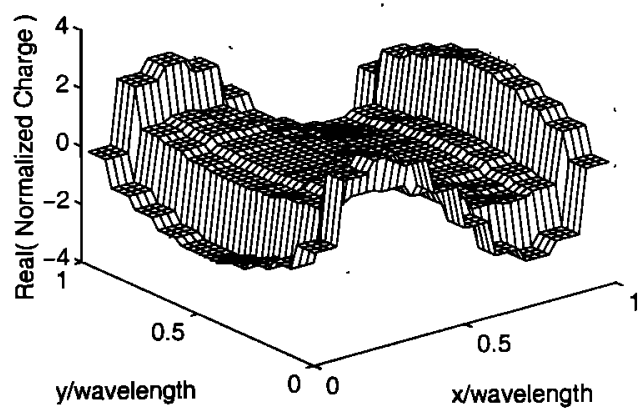

(c)

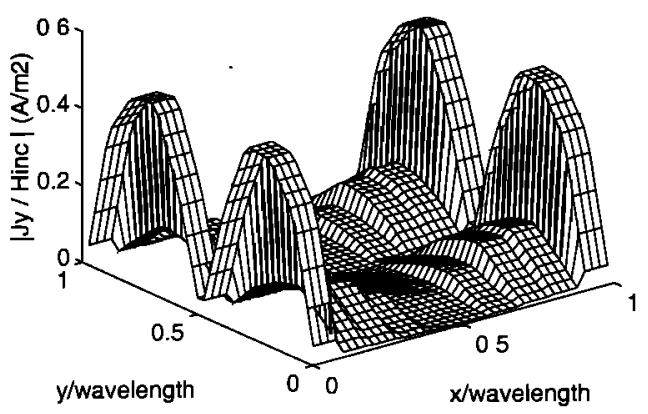

(b)

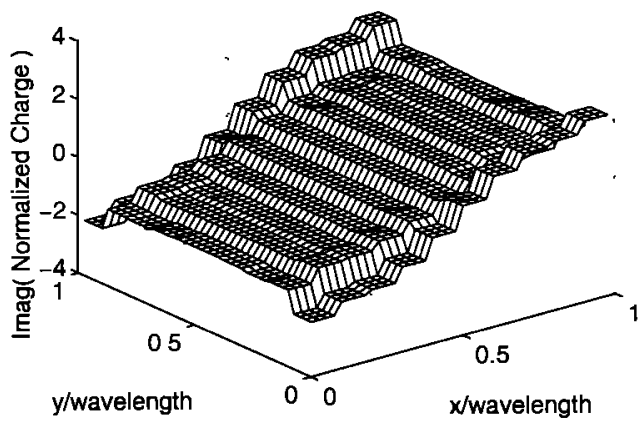

(d)

Figure 3. Normalized induced current and charge distributions on the $\lambda \times \lambda$ patch obtained using the RT BFs: (a) Magnitude of copolar current $\left(J_{x}\right)$, (b) magnitude of cross-polar current $\left(J_{y}\right)$, (c) real part of the charge, and (d) imaginary part of the charge.

For a proper PW constant modeling of the charge, we must have

$$
E_{\text {int }} \geq F
$$

which can be converted to

$$
V_{\text {int }} \geq \chi
$$

using (7)-(10) and to

$$
2 E_{\text {int }} \geq E_{\text {ext }}
$$

using (11). Both (16) and (17) can easily be satisfied for open and closed surfaces. Thus the use of RT BFs to approximate the current distribution does not pose a problem for the $\mathrm{PW}$ constant modeling of the charge distribution. In order to demonstrate this,
Figure 3 shows the magnitudes of the $x$ and $y$ components of the current distribution and the real and imaginary parts of the charge distribution induced on a $\lambda \times \lambda$ perfect electric conductor (PEC) patch on the $x-y$ plane. (The results showing the real and imaginary parts of the charge distribution are scaled by a factor of $i \omega$. Therefore the real and imaginary parts may be interchanged, if desired.) The patch is illuminated by a normally incident plane wave, whose electric field is polarized in the $x$ direction. Each side of the patch is divided into 10 subsections so that the total number of RT BFs is $E_{\text {int }}=180$ and the charge distribution is approximated by $F=100$ constantcharge plateaus. The results presented in Figure 3 are as smooth as they can be with the given discretization and with the PW linear and PW constant modeling of the current and the charge, respectively. 


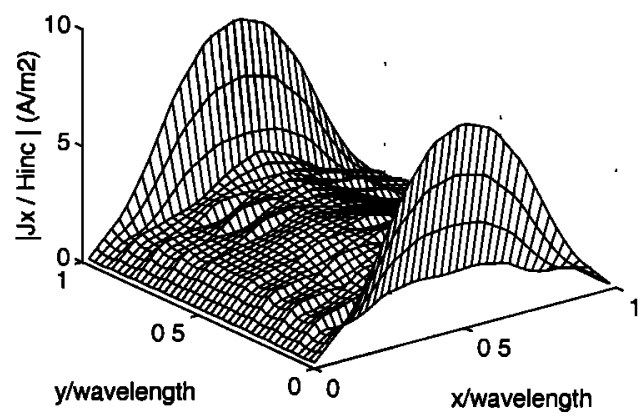

(a)

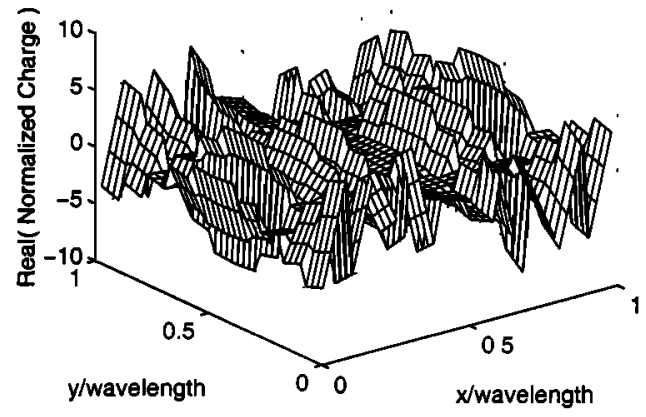

(c)

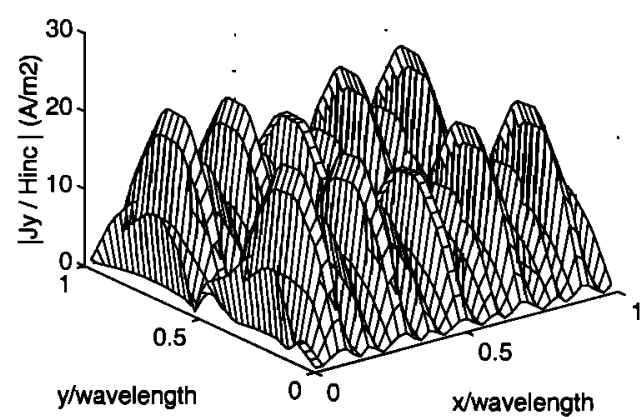

(b)

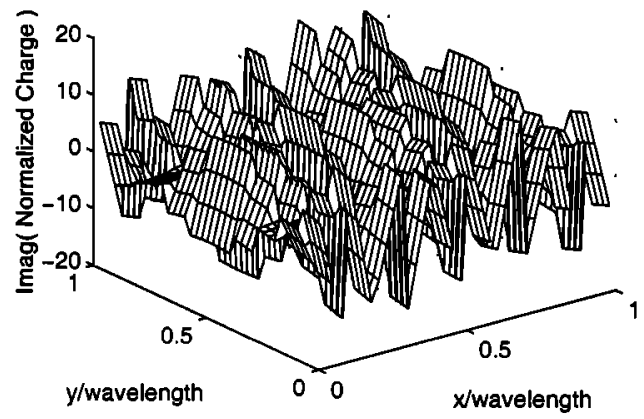

(d)

Figure 4. Normalized induced current and charge distributions on the $\lambda \times \lambda$ patch obtained using the higher-order RT BFs: (a) Magnitude of copolar current $\left(J_{x}\right)$, (b) magnitude of cross-polar current $\left(J_{y}\right)$, (c) real part of the charge, and (d) imaginary part of the charge.

\subsection{Higher-Order Rooftop Basis Functions}

These BFs can be obtained from a straightforward extension of the regular RT BFs. The higher-order RT BFs considered here are slightly different from the regular RT BFs in such a way that although the current approximation is PW linear and continuous along the direction of the current flow as in regular RT BFs, it is PW linear and discontinuous in the transverse direction, as shown in Figure 1b as opposed to the PW constant (and discontinuous) approximation of the regular RT BFs. Sercu et al. [1994] called these types of BFs "first-order" RT BFs and proposed to use them on planar structures.

We used these BFs in the solution of the scattering problem outlined in the previous section. The current and charge distributions are shown in Figure 4 in the same format as in Figure 3. The copolar induced current in Figure 4a seems to have the correct distribution and the proper linear variation in the transverse direction as supported by these higher-order RT BFs. These BFs model both components of the current with a distribution of $\mathrm{PW}$ bilinear functions on rectangular subdomains. As long as the approximated current is continuous along the direction of its flow, this type of PW bilinear modeling appears to be perfectly valid. However, the cross-polar component of the current and the charge distribution are unmistakably incorrect, as seen in Figures $4 \mathrm{~b}-4 \mathrm{~d}$. What can be the reason for the failure of these seemingly legitimate $\mathrm{BFs}$ in the solution of this simple scattering problem?

The answer to the above question may be sought in the way these BFs model the charge distribution. 
Since the current is approximated by the bilinear forms

$$
\begin{aligned}
& J_{x}(x, y)=A_{x} x y+B_{x} x+C_{x} y+D_{x} \\
& J_{y}(x, y)=A_{y} x y+B_{y} x+C_{y} y+D_{y}
\end{aligned}
$$

on each rectangular patch, the charge is approximated by

$$
\rho(x, y)=A_{x} y+A_{y} x+\left(B_{x}+C_{y}\right),
$$

which is linear in both $x$ and $y$. This approximation requires three DOFs per rectangle, which puts the total number of the DOFs required for the charge approximation at $3 F$. On the other hand, the number of the supplied DOFs is $2 E_{\text {int }}$, since the higher-order RT BFs are defined only on the internal edges and with two DOFs per edge. Then, for a proper PW linear (in both $x$ and $y$ ) modeling of the charge, we must have

$$
2 E_{\text {int }} \geq 3 F \text {. }
$$

We caution the reader that the above will turn out to be a necessary but not sufficient condition at the end of this section. However, we will continue as in the previous section to reach that conclusion. Using (11), equation (21) can be expressed as

$$
2 E_{\text {int }} \geq 3 E_{\text {ext }},
$$

which is always satisfied on closed surfaces, since $E_{\text {ext }}=0$. However, the situation is more interesting for open surfaces, since whether (22) is satisfied or not depends on the type of the mesh used on the surface. As an example, consider the patch problem again. If the patch is meshed into $2 \times N$ (where $N$ is any positive integer), $3 \times 3$, or $3 \times 4$ rectangles, then (22) is not satisfied, but $3 \times 5,4 \times 4$, or denser meshes satisfy (22). It is even more interesting to note that the results presented in Figure 4 are obtained with a $10 \times 10$ mesh, which should satisfy (22). Therefore we conclude that merely counting the DOFs required by the charge approximation may not be sufficient; the types of the DOFs supplied by the current approximation and required by the charge approximation should also match. We will explain this constraint with the help of Figure 5, where a $4 \times M$ ( $M \geq 4$ ) meshing of a patch is depicted. In Figure $5 \mathrm{a}$, the BFs are indicated on the edges and the charge subdomains are numbered using parentheses.

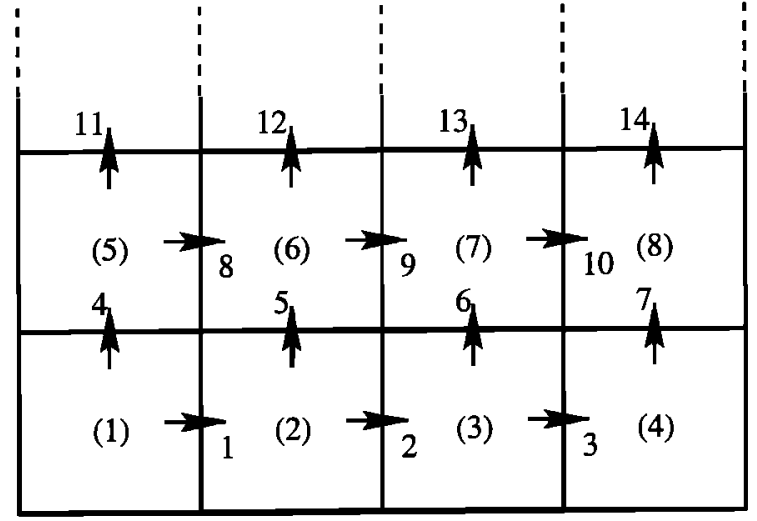

(a)

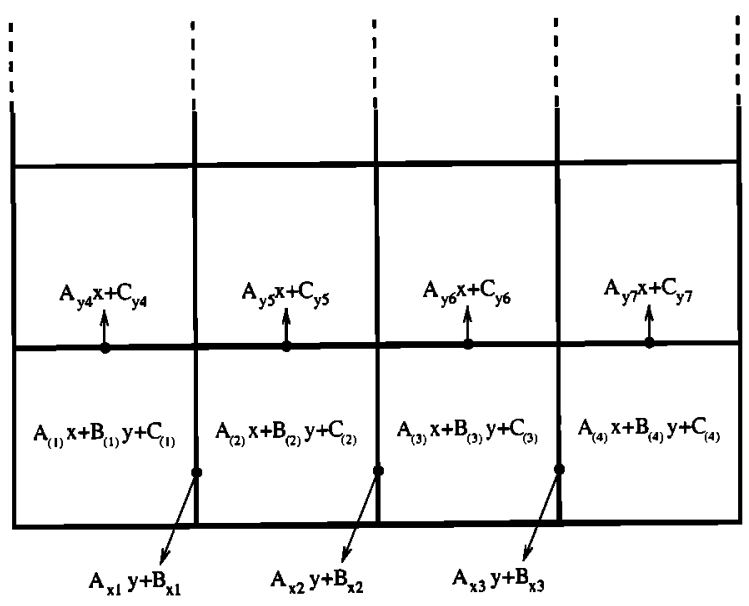

(b)

Figure 5. (a) Arrows denote the current BFs defined on the edges. Numbers in parentheses denote the subdomains on which piecewise (PW) linear approximation of the charge is obtained. (b) Required charge degrees of freedom (DOFs) are shown in the subdomains. Charge DOFs supplied by the current BFs are shown on the edges.

Let us focus on the first four subdomains that are surrounded by the first seven BFs. As depicted in Figure $5 \mathrm{~b}$, proper approximation of the charge on these four subdomains requires 12 DOFs: four linear variations in $x$, four linear variations in $y$, and four constant plateaus. The surrounding seven BFs supply 14 DOFs for the current approximation: four linear variations in $x$, three linear variations in $y$, and seven constant values. Therefore, although the total 


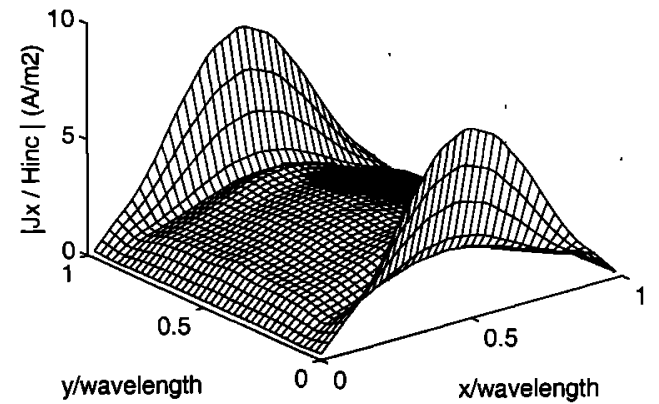

(a)

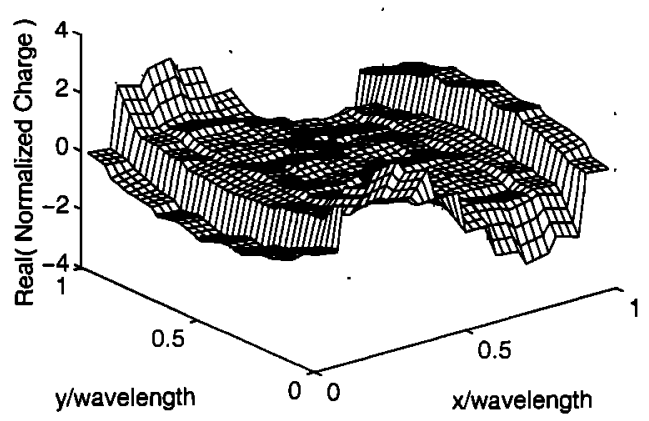

(c)

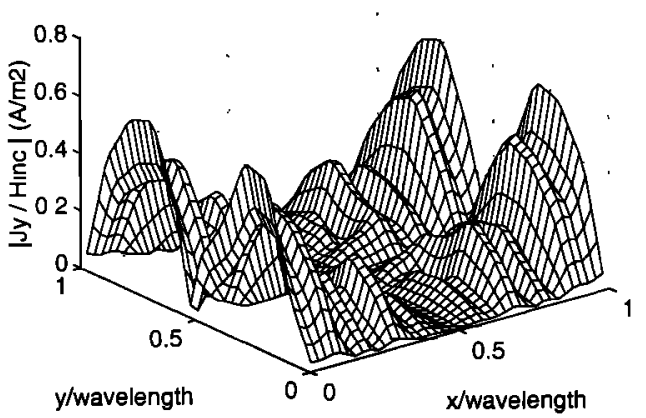

(b)

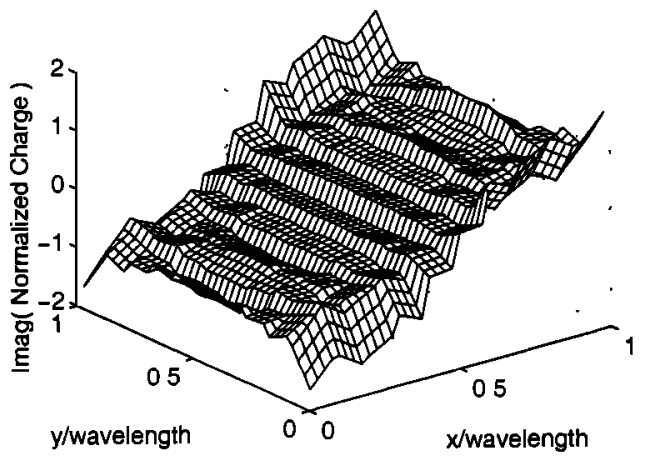

(d)

Figure 6. Normalized induced current and charge distributions on the $\lambda \times \lambda$ patch obtained using the transversely continuous higher-order RT BFs: (a) Magnitude of copolar current $\left(J_{x}\right)$, (b) magnitude of cross-polar current $\left(J_{y}\right),(\mathrm{c})$ real part of the charge, and (d) imaginary part of the charge.

number of the DOFs supplied by the current BFs seems to be sufficient $(14>12)$, when these DOFs are broken down to specific types, not all of the necessary DOFs for a proper approximation of the charge are available. In this example, the charge needs four linear DOFs in $y$, whereas the current BFs can supply only three. This conclusion can be extended to other cases of open-surface meshings, too. We do not envision such a problem for closed surfaces.

The reason why the cross-polar component of the current shown in Figure $4 \mathrm{~b}$ is grossly incorrect while the copolar current in Figure $4 \mathrm{a}$ is slightly in error is not well understood. However, we confirmed by further numerical experiments that increasing the mesh density and hence the number of BFs did not reduce the error in the cross-polar current.

In summary, the higher-order RT BFs considered in this subsection turned out to be genuinely interesting since they appeared to be perfectly legitimate candidates for current modeling, but then we showed that the condition (22) is not satisfied for all open surfaces, and finally we showed that even compliance with (22) is not sufficient for most open surfaces (exceptions are possible). Therefore this type of BF should be employed with great care. It should be noted that although Sercu et al. [1994] suggested the use of these BFs, they did not present any results obtained with them. 


\subsection{Transversely Continuous Higher-Order Rooftop Basis Functions}

These basis functions can be obtained from the higher-order RT BFs of the previous section by constraining the current to be continuous in the transverse direction in addition to the direction of flow, as shown in Figure 1c. These constraints are implemented on the internal nodes of a surface for both components of the current. Thus the total number of DOFs supplied by these current BFs drops from $2 E_{\text {int }}$ to $2 E_{\text {int }}-2 V_{\text {int }}$. Both the current and the charge are still modeled by $\mathrm{PW}$ bilinear and $\mathrm{PW}$ linear (in $x$ and $y$ ), respectively, approximations on rectangular subdomains as given by (19) and (20). Thus the charge approximation requires $3 F$ DOFs. Then, for a correct modeling of the charge, the condition

$$
2 E_{\text {int }}-2 V_{\text {int }} \geq 3 F
$$

must be satisfied. Using (7)-(10), equation (23) can be expressed as

$$
-2 \chi \geq F
$$

which is a contradiction for both open and closed surfaces. Therefore, although the current-modeling capabilities of these BFs seem to be flawless, we can expect their inability to properly model the charge distribution to contaminate the solution.

Figure 6 shows the current and charge distributions obtained on a $\lambda \times \lambda$ patch for the same scattering problem as before. Although the copolar current in Figure 6a seems to have a fairly correct distribution, the same cannot be said for the cross-polar component of the current and the charge distribution, shown in Figures $6 \mathrm{~b}-6 \mathrm{~d}$.

\subsection{Pyramidal Basis Functions with Rectangular Bases}

These BFs can be classified as "nodal" BFs since the heights of the pyramids are associated with the values of the unknown function at every node of the surface, as shown in Figure 1d. The use of these BFs results in PW bilinear approximation of the current on rectangular subdomains. Note that this is the same type of approximation obtained with the higher-order RT BFs. Indeed, the pyramidal BFs with rectangular bases are completely equivalent to transversely continuous higher-order RT BFs. Be- cause of this equivalence, we should obtain the same current and charge results as in the previous section.

Consider a closed surface, such as the rectangular prism shown in Figure 2. The total number of supplied DOFs is $2 \mathrm{~V}$ since two pyramids are defined per node for the two components of the surface current. A correct $\mathrm{PW}$ linear (in two variables) modeling of the charge requires

$$
2 V \geq 3 F .
$$

Using $\chi=2, \quad E_{\text {ext }}=0$, and $E=2 F$, we arrive at

$$
V \leq 6 \text {, }
$$

which cannot be satisfied by any meaningful geometry. Thus these BFs are not capable of modeling the charge properly. The same conclusion holds for open surfaces, too. Considering the patch geometry of Figure 2c, it is noted that although the required number of DOFs for the charge distribution is still $3 F$, the number of available DOFs drops from $2 V$ to $2 V_{\text {int }}+V_{\text {ext }}-4$ since no pyramidal BFs are defined at the edges of the patch for the current component normal to that edge.

\subsection{Pyramidal Basis Functions with Triangular Bases}

These nodal BFs are defined on a collection of triangular subdomains with a common vertex, as shown in Figure 1e. The current approximation is linear in $x$, linear in $y$, or linear in both $x$ and $y$ on each subdomain. Therefore the charge is approximated by a PW constant distribution.

Let us first consider a closed surface, such as the rectangular prism shown in Figure 2b. Because two of these pyramidal BFs are used at each node to model the two components of the surface current and because one constant-height plateau per subdomain is required for the PW constant modeling of the charge, we must have

$$
2 V \geq F \text {. }
$$

Using $\chi=2, \quad E_{\text {ext }}=0$, and $2 E=3 F$, we find that (27) is always satisfied and that this type of pyramidal current BFs can flawlessly model both the current and the charge on closed surfaces.

On the other hand, the situation for open surfaces is a little different since no BFs are defined at the 


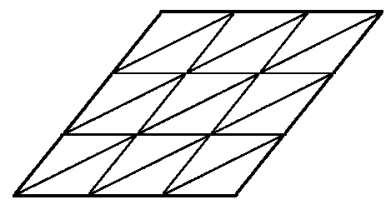

(a)

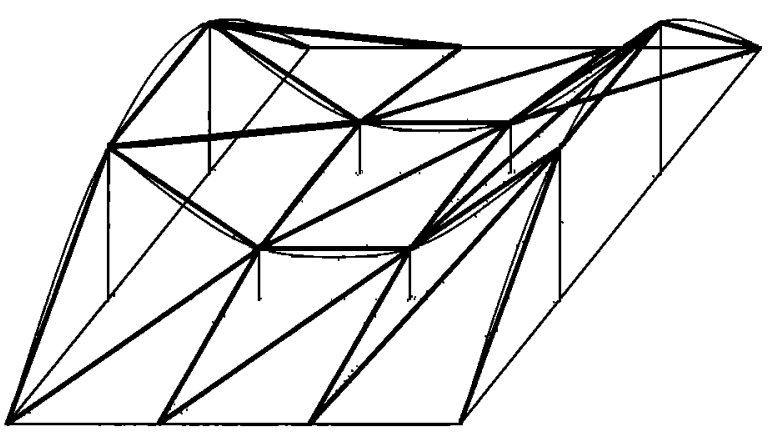

(c)

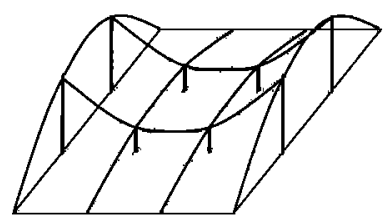

(b) imation of each component of the surface current on the patch, as depicted in Figure 7. These types of pyramidal BFs are very well known. Houshmand et al. [1991] presented their Fourier transforms in order to extend their use to spectral-domain formulations. However, considering the current alone is not sufficient, one should also pay attention to how the charge is modeled by the divergence of the current BFs. Furthermore, whether one type of BFs is "good" or not also depends on the specific surface and meshing.

\subsection{Rao-Wilton-Glisson Basis Functions}

These famous "vector" BFs, which we will refer to as RWG BFs, are first introduced by Rao et al. [1982]. RWG BFs are defined on the interior edges of a surface, and they approximate the current with a PW linear distribution as shown in Figure 1f. Consequently, the charge is approximated by a $\mathrm{PW}$ constant distribution. RWG BFs are often called "RT BFs on triangular subdomains" due to their resemblance to the RT BFs. In agreement with this observation, the condition for the RWG BFs to properly model the charge distribution is

$$
E_{\text {int }} \geq F,
$$

which is the same as (15) for the RT BFs. Using (7) $-(10)$, this condition can be converted to

$$
V_{\text {int }} \geq \chi
$$

boundaries of an open surface for the current component normal to that boundary. Then, the total number of DOFs supplied by the current approximation is less than $2 \mathrm{~V}$ and it is not certain any more that the required number of DOFs for a proper charge approximation is available. For instance, let us consider the rectangular patch in Figure 2b. The number of available DOFs drops from $2 V$ to $2 V_{\text {int }}+V_{\text {ext }}-4$, and therefore, for a proper PW constant charge approximation, we must have

$$
2 V_{\text {int }}+V_{\text {ext }}-4 \geq F .
$$

Using (7) and (14) together with $\chi=1$ for this surface, we can show that (28) is a contradiction, which means that these BFs cannot simultaneously model both the current and the charge distributions on an open surface such as a rectangular patch. We confirmed this conclusion with numerical experiments, too.

This is another interesting result since one would expect to be able to have a correct PW linear approx- and using (13), it can be converted to

$$
E_{\text {int }} \geq E_{\text {ext }}
$$

both of which can easily be satisfied by open $(\chi=1)$ and closed $\left(E_{\text {ext }}=0\right)$ surfaces.

Thus we conclude that RWG BFs can be safely used on arbitrary triangulations of both open and closed surfaces. Figure 8 shows the current and charge distributions on the $\lambda \times \lambda$ patch obtained by employing the RWG BFs in the solution of the scattering problem outlined earlier. The results are as good as they can be with this discretization. Note that there is a remarkable difference between the magnitudes of the copolar and cross-polar currents, i.e., $J_{x}$ is significantly dominant compared to $J_{y}$. Since RWG BFs are vector BFs, the same set of RWG BFs are employed to model both $J_{x}$ and $J_{y}$. This explains the nonsmooth behavior of the cross-polar current shown in Figure 8b. This behavior does not contaminate the charge distribution as seen in Fig- 


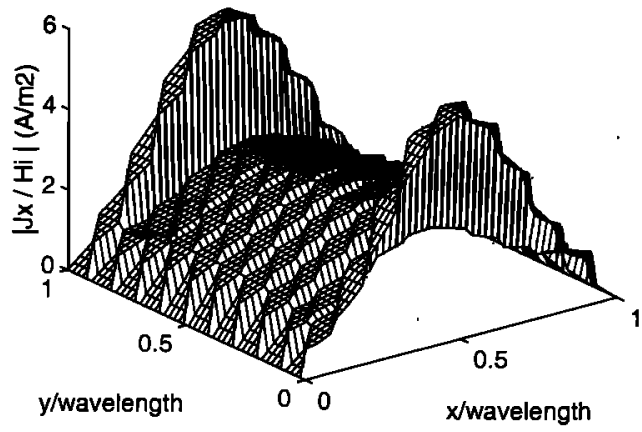

(a)

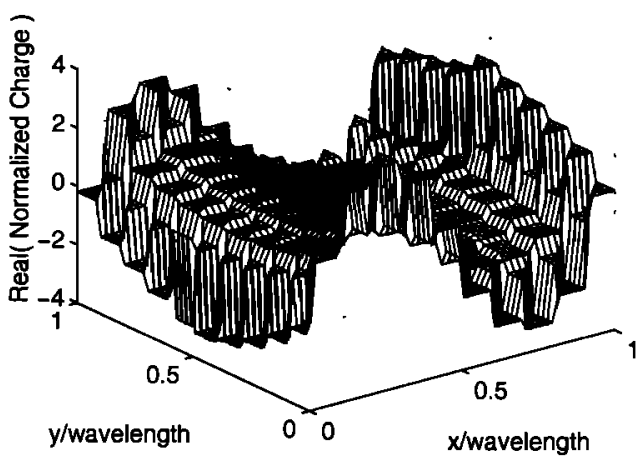

(c)

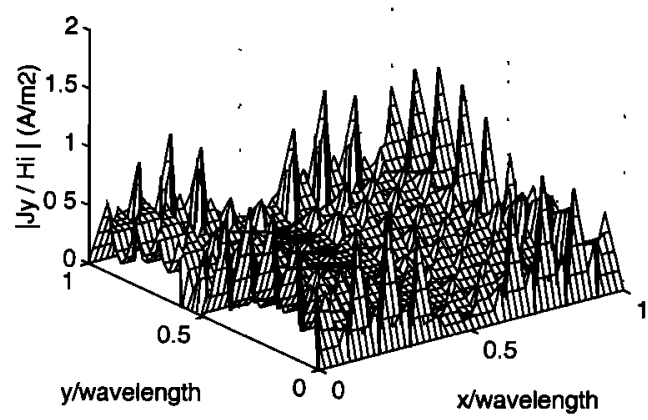

(b)

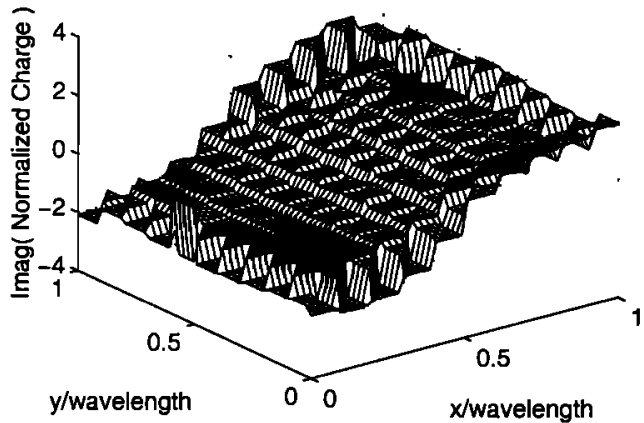

(d)

Figure 8. Normalized induced current and charge distributions on the $\lambda \times \lambda$ patch obtained using the RWG BFs: (a) Magnitude of copolar current $\left(J_{x}\right)$, (b) magnitude of cross-polar current $\left(J_{y}\right),(\mathrm{c})$ real part of the charge, and (d) imaginary part of the charge.

ures $8 \mathrm{c}$ and $8 \mathrm{~d}$, and thus this is the correct solution that can be obtained with the use of the RWG BFs on this discretization. If the polarization of the incident electric field is modified to be in the $\hat{x}+\hat{y}$ direction, then the magnitudes of $J_{x}$ and $J_{y}$ are in the same order and we obtain similar smooth distributions for both of them.

\subsection{RWG Basis Functions Augmented With Magnetic Basis Functions}

In the solution of electromagnetic problems involving magnetic current distributions, BFs that are different from the electric current BFs are usually employed. Several such "magnetic" BFs with triangular subdomains are developed and available in the liter- ature. In addition to using these magnetic BFs to model the magnetic current, Wandzura [1992] proposed augmenting the RWG BFs with magnetic BFs to model the electric current distribution. He suggested this combination in order to obtain an electric current distribution with a higher level of continuity, and thus better modeling. The level of continuity and thus the quality of the approximation can be further improved by adding higher-order electric and magnetic BFs.

Sarkar et al. [1990] proposed using

$$
\mathbf{b}_{\text {MAG }}(\mathbf{r})=\hat{n} \times \mathbf{b}_{\mathrm{RWG}}(\mathbf{r})
$$

as a magnetic $B F$, where $b_{R W G}(r)$ is the RWG $B F$ and $\hat{n}$ is the unit vector normal to the surface con- 
taining both of the RWG BFs. These BFs cause magnetic line charge accumulation along the edges of the triangular subdomains since they render the normal component of the current discontinuous at these edges. Therefore these BFs will not be considered here.

Two other magnetic BFs, one proposed by Jacobus and Landstorfer [1993] and the other proposed by Wandzura [1992], do not have the above problem. These two magnetic BFs are very similar in many respects, especially in the way they model the current, but are not exactly the same. Perhaps their most significant difference is in the way they model the charge distribution. The divergence of Wandzura's magnetic BF is identically equal to zero, which means that it does not attempt to model the charge at all. Surprisingly, this will turn out to be a desired feature that will be discussed at the end of this section.

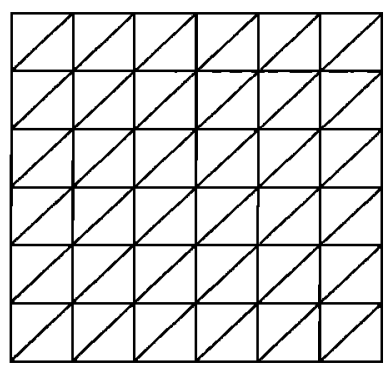

(a)

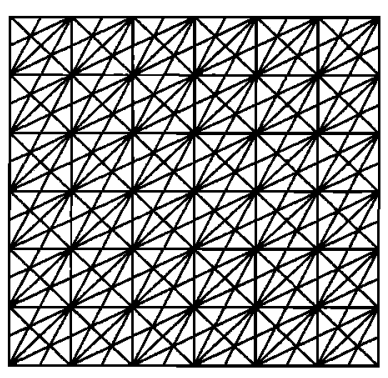

(b)

Figure 9. (a) Triangular subdomains for PW constant current modeling using the RWG BFs. (b) Sixtimes denser triangular meshing for the PW constant current obtained when the RWG BFs are augmented with the magnetic BFs.

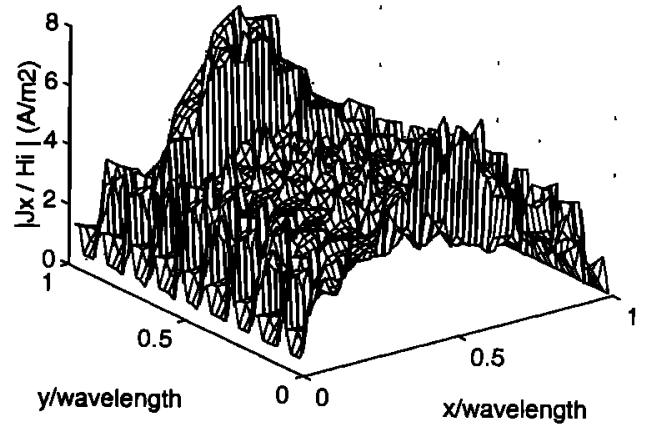

(a)

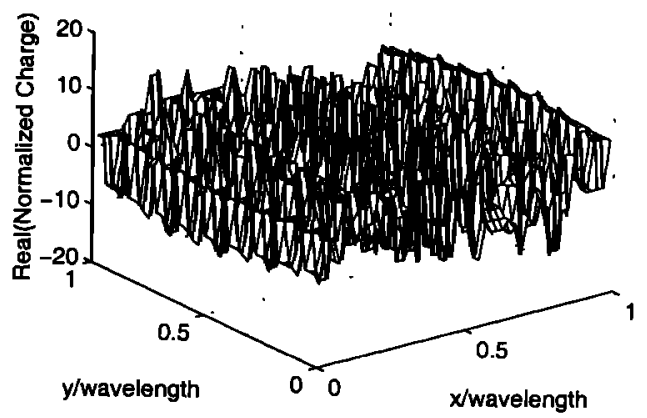

(c)

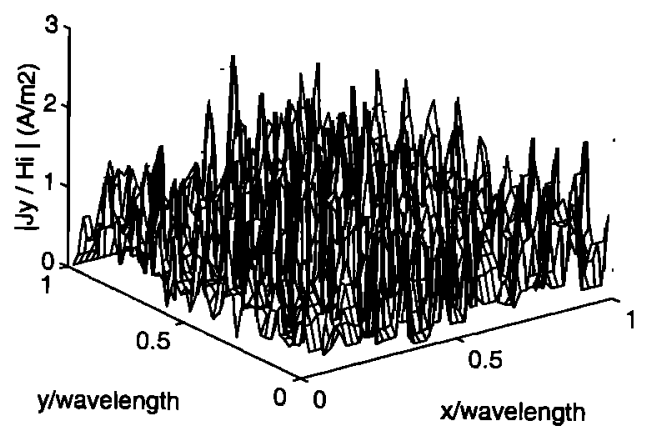

(b)

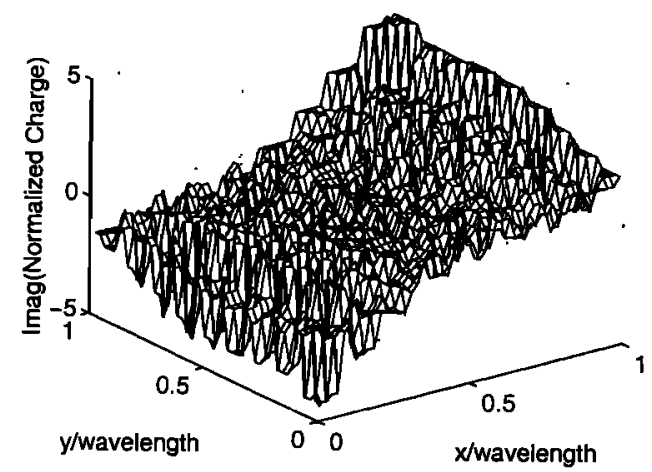

(d)

Figure 10. Normalized induced current and charge distributions on the $\lambda \times \lambda$ patch obtained using the RWG BFs augmented with the magnetic BFs: (a) Magnitude of copolar current $\left(J_{x}\right)$, (b) magnitude of cross-polar current $\left(J_{y}\right)$, (c) real part of the charge, and (d) imaginary part of the charge. 
The magnetic BF proposed by Jakobus and Landstorfer [1993] is actually a combination of two RWG BFs arranged to be approximately orthogonal to a regular RWG BF as shown in Figure 1g. Therefore the divergence of this magnetic $\mathrm{BF}$ is a combination of four constant-charge plateaus on triangular subdomains that are half of regular triangular subdomains. This situation arises when only a single magnetic BF is considered. When all of the magnetic BFs associated with all edges are considered, the charge needs to be approximated by six PW constant plateaus on each triangular subdomain, as shown in Figure 9. In contrast, the original RWG BFs require one constant charge plateau on each triangular subdomain.

When the RWG BFs are augmented with Jakobus and Landstorfer's [1993] magnetic BFs, the total number of the DOFs is equal to $E_{\text {int }}+E$, since the RWG BFs are defined only on the internal edges, whereas the magnetic BFs can be used on all edges including the external edges. The number of the DOFs required for a proper PW constant charge approximation is $6 F$. Then, we must have

$$
E_{\text {int }}+E \geq 6 F \text {, }
$$

which is impossible since $E_{\text {int }}+E=2 E_{\text {int }}+E_{\text {ext }}=3 F$ using (9) and (13). Therefore we conclude that these BFs cannot be used to augment RWG BFs. This conclusion is supported by the current and charge results shown in Figure 10. These results are obtained on the $\lambda \times \lambda$ patch using the combination of RWG and magnetic BFs for the scattering problem outlined earlier.

A similar problem is not expected when Wandzura's [1992] magnetic BFs are used to augment the RWG BFs. This is because the divergence of Wandzura's magnetic BF is identically equal to zero and thus does not increase the number of the DOFs required by the charge distribution. Then, the condition for a proper PW constant charge distribution becomes

$$
E_{\text {int }}+E \geq F,
$$

which is always satisfied for both open and closed surfaces.

\section{Conclusions}

In this paper, we have clearly demonstrated the need to consider, in addition to other constraints documented in the literature, how well the charge distribution is satisfied when choosing a set of BFs to approximate the surface electric current. Some commonly used BFs, such as the RT and RWG BFs, satisfy this constraint unconditionally and therefore can be safely used to model both the current and the charge distributions. This point has been rigorously proven by analyzing the topology of open and closed surfaces meshed into networks of triangles and quadrangles. On the other hand, surprising results emerged from the investigation of some other BFs. Among these are higher-order RT and pyramidal BFs, which seem to be perfect candidates to support $\mathrm{PW}$ bilinear and $\mathrm{PW}$ linear approximations of the current distribution. However, we have rigorously proven and computationally demonstrated that these BFs do not always supply a sufficient number of DOFs for a consistent charge model. We have also proven and demonstrated that the use of some magnetic and/or higher-order BFs to improve the quality of the electric current approximation may have disastrous results, whereas some others may serve the intended purpose.

Granted, not every possible BF can be analyzed in a document of this size. It is not merely the results of a class of sample BFs presented in section 4 that we intend to pass on to the readers, but also the idea that the current BFs should be chosen with an eye on how well they model the charge distribution, in addition to the current distribution.

Acknowledgments. The authors acknowledge two anonymous reviewers for their meticulous reviews, which significantly improved the manuscript.

\section{References}

Aberegg, K. R., A. Taguchi, and A. F. Peterson, Application of higher-order vector basis functions to surface integral equation formulations, Radio Sci., 31(5), 1207-1213, 1996.

Aksun, M. I., and R. Mittra, Choices of expansion and testing functions for the method of moments applied to a class of electromagnetic problems, IEEE Trans. Microwave Theory Tech., MTT41(3), 503-509, 1993.

Andersson, T., Moment-method calculations of scattering by a square plate using singular basis functions and multipole expansions, J. Electromagn. Waves Appl., 7(1), 93-121, 1993.

Canning, F. X., Improved impedance matrix local- 
ization method, IEEE Trans. Antennas Propag., AP-41(5), 659-667, 1993.

Chew, W. C., L. Gürel, Y. M. Wang, G. Otto, R. Wagner, and Q. H. Liu, A generalized recursive algorithm for wave-scattering solutions in two dimensions, IEEE Trans. Microwave Theory Tech., MTT-40(4), 716-723, 1992.

Coifman, R., V. Rokhlin, and S. Wandzura, The fast multipole method for the wave equation: A pedestrian prescription, IEEE Antennas Propag. Mag., 35(3), 7-12, 1993.

Glisson, A. W., and D. R. Wilton, Simple and efficient numerical methods for problems of electromagnetic radiation and scattering from surfaces, IEEE Trans. Antennas Propag., AP-28(5), 593$603,1980^{\circ}$

Graglia, R. D., D. R. Wilton, and A. F. Peterson, Higher order interpolatory vector bases for computational electromagnetics, IEEE Trans. Antennas Propag., AP-45(3), 329-342, 1997.

Gürel, L., and M. I. Aksun, Electromagnetic scattering solution of conducting strips in layered media using the fast multipole method, IEEE Microwave Guided Wave Lett., 6(8), 277-279, 1996.

Gürel L., and W. C. Chew, Recursive T-matrix algorithms with reduced complexities for scattering from three-dimensional patch geometries, IEEE Trans. Antennas Propag., AP-41(1), 91-99, 1993.

Harrington R. F., Field Computation by Moment Methods, Krieger, Melbourne, Fla., 1982.

Houshmand, B., W. C. Chew, and S. W. Lee, Fourier transform of a linear distribution with triangular support and its application in electromagnetics, IEEE Trans. Antennas Propag., AP-39(2), 252254, 1991.

Jakobus, U., and F. M. Landstorfer, Novel basis function for the equivalent magnetic current in the method of moments solution of dielectric scattering problems, Electron. Lett., 29(14), 1272-1273, 1993.

Kim, H., H. Ling, and C. Lee, A fast moment method algorithm using spectral domain wavelet concepts, Radio Sci.31(5), 1253-1261, 1996.

Kolundžija, B.M., and B. D. Popović, Entire-domain Galerkin method for analysis of metallic antennas and scatterers, IEE Proc., Part H, 140(1), 1-10, 1993.

Lu, C. C., and W. C. Chew, Fast algorithm for solving hybrid integral equations, IEE Proc., Part $H$, 140(6), 455-460, 1993.
Miller, E. K., L. Medgyesi-Mitschang, and E. H. Newman (Eds.), Computational Electromagnetics, Inst. of Electr. and Electron. Eng., New York, 1992.

Mortenson, M. E., Geometric Modeling, John Wiley, New York, 1985.

Peterson, A. F., and K. R. Aberegg, Parametric mapping of vector basis functions for surface integral equation formulations, Appl. Comput. Electromagn. Soc. J., 10(3), 107-115, 1995.

Rao, S. M., D. R. Wilton, and A. W. Glisson, Electromagnetic scattering by surfaces of arbitrary shape, IEEE Trans. Antennas Propag., AP-30(5), 409-418, 1982.

Richmond, J. H., On the variational aspects of the moment method, IEEE Trans. Antennas Propag., AP-39(4), 473-479, 1991.

Rokhlin, V., Rapid solution of integral equations of scattering theory in two dimensions, J. Comput. Phys., 86(2), 414-439, 1990.

Rubin, B. J., and S. Daijavad, Radiation and scattering from structures involving finite-size dielectric regions, IEEE Trans. Antennas Propag., AP38(11), 1863-1873, 1990.

Sarkar, T. K., A note on the choice of weighting functions in the method of moments, IEEE Trans. Antennas Propag., AP-33(4), 436-441, 1985.

Sarkar, T. K., From "reaction concept" to "conjugate gradient": Have we made any progress?, IEEE Antennas Propag. Soc. Newsl., 31, pp. 612, Aug. 1989.

Sarkar, T. K., A. R. Djordjević, and E. Arvas, On the choice of expansion and weighting functions in the numerical solution of operator equations, IEEE Trans. Antennas Propag., AP-33(9), 988996, 1985.

Sarkar, T. K., S. M. Rao, and A. R. Djordjević, Electromagnetic scattering and radiation from finite microstrip structures, IEEE Trans. Microwave Theory Tech., MTT-38(11), 1568-1575, 1990.

Sercu, J., N. Faché, and P. Lagasse, First-order rooftop functions for the current discretisation in the method of moments solution of planar structures, in Proceedings of the IEEE Antennas Propagat. Society International Symposium, pp. 21702173, Inst. of Electr. and Electron. Eng., New York, 1994.

Song, J. M., and W. C. Chew, Moment method solutions using parametric geometry, J. Electromagn. Waves Appl., 9(1/2), 71-83, 1995. 
Valle, L., F. Rivas, and M. F. Cátedra, Combining the moment method with geometrical modelling by NURBS surfaces and Bézier patches, IEEE Trans. Antennas Propag., AP-38(3), 373381, 1994.

Wandzura, S., Electric current basis functions for curved surfaces, Electromagnetics, 12, 77-91, 1992.

L. Gürel, Department of Electrical and Electronics Engineering, Bilkent University, TR-06533, Bilkent, Ankara, Turkey. (lgurel@ee.bilkent.edu.tr)
I.K. Şendur, Electroscience Laboratory, Ohio State University, 1320 Kinnear Road, Columbus, OH 43212. (senduri@ ee.eng.ohio-state.edu)

K. Sertel, Radiation Laboratory, University of Michigan, 1231 Beal Avenue, Ann Arbor, MI 48109. (ksertel@engin.umich.edu)

(Received May 27, 1998; revised October 15, 1998; accepted January 25, 1999.) 